\title{
Prescribing antidepressants for depression: time to be dimensional and inclusive
}

Ian M Anderson and Peter M Haddad

\begin{abstract}
The article by Middleton and Moncrieff questions the role of antidepressants in treating depression on both philosophical and practical grounds; namely that depression isn't a brain disease to be treated by a drug and that antidepressants are ineffective except as placebos. We argue that their stance is unhelpful and factually incorrect and that a more dimensional and integrative approach is needed in order to be able to best tailor treatment to individual needs. This involves a personalised assessment of the likely benefits and risks of both psychological and drug approaches when recommending treatment for someone with depression. Keywords

antidepressants; depression; placebo; psychological treatment; randomised controlled trials.
\end{abstract}

\section{INTRODUCTION}

To caricature, if only slightly, the position of Middleton and Moncrieff, ${ }^{1}$ the case is put forward for 'antidepressants bad, psychological treatment good' based on two main arguments. First, that the use of antidepressants is based on the erroneous medical model that 'depression is a reversible, or partly reversible, brain disease that can usefully be treated by drugs', and secondly that antidepressants work through the placebo effect and therefore their risks outweigh their benefits. We believe their case is based on both dualistic and categorical errors in reasoning, and on misrepresentation of the evidence.

\section{AVOIDING MIND-BRAIN DUALISM}

Applying a Cartesian dualistic approach, if depression is not a disease of the brain, it must be one of the mind, which can only be treated by approaches that address the 'stuff' of the mind, that is, consciousness, understanding, and meaning. The use of 'disease' in this context implies a categorical distinction from 'health', rather than the continuum we recognise with depression in terms of symptom number and severity, and duration and degree of functional impairment. ${ }^{2}$ If we take a different approach, that neural circuitry underpins our mood and consciousness, then we can see how events that affect our 'mind' also result in brain changes and how alterations in brain activity can have conscious or mood counterparts. Empirical evidence for this is now overwhelming. To give but a few examples: selfinduced temporary low mood in healthy volunteers causes changes in brain activity that are very similar to those seen in those with a diagnosis of depression, ${ }^{3}$ arguing for specific brain circuits being involved in the experience of low mood. Manipulations of brain serotonin availability, including with antidepressants, influence how the brain processes emotional material, ${ }^{4}$ and individuals with a history of depression experience recurrence of depressed mood when brain serotonin is lowered, with associated changes in brain function. ${ }^{5}$

The involvement of brain circuits and neurotransmitter function in depressed mood is therefore difficult to deny, as is the potential for 
influencing these with drugs - or indeed with psychotherapy, self-help, or change in life circumstances. This is a different, more meaningful, and more inclusive formulation of the relationship between depression and the brain than the term 'brain disease' set up as an 'Aunt Sally' by Middleton and Moncrieff. ${ }^{1}$ We agree, however, that it is important to avoid using this term as a careless shorthand that can reinforce an 'either-or' mentality about causation and treatment.

\section{ANTIDEPRESSANTS WORK BUT NEED TO BE TARGETED}

The second argument that antidepressants just don't work flies in the face of the general agreement, even by detractors, that there are drug-placebo differences that are small in mild to moderate depression and increase with severity. ${ }^{6-8}$ This change in effect size with depression severity simply cannot be explained by a placebo effect, nor can the robust evidence that antidepressants are highly effective in preventing relapse. ${ }^{9}$ The relevant questions then become 'How well do they work?' and 'How useful are they?'. In the updated National Institute for Health and Clinical Excellence (NICE) guidance on treating depression, ${ }^{2}$ the concept of a discrete cut-off for a clinically important size of effect (a categorical distinction) is dropped; a moment's thought makes it clear that a more dimensional approach is needed. If, for argument's sake, the cut-off is 3 points on the Hamilton Depression Rating Scale, does that mean that 2.9 points is worthless but 3.1 points beneficial? And who decides on this threshold? Clinical context is important, and relatively small effects might be worthwhile for treating a disorder with potentially serious outcomes, a high probability of not improving spontaneously, or non-response to previous treatments. A dimensional approach allows flexibility in applying a risk-benefit decision to individual cases. For example, although antidepressants appear to offer little benefit compared with placebo in recent-onset mild depression, they do have a moderate effect size in dysthymia (chronic mild depression). ${ }^{1}$ Therefore, in a situation where milder degrees of depression are longstanding, or persist after other approaches have not produced benefit, should patients be denied a trial of antidepressants because of a dogmatic view that they 'don't work' in mild depression?

\section{BALANCING THE RISKS AND BENEFITS OF TREATMENT}

Where we can agree with Middleton and Moncrieff ${ }^{1}$ is that all treatments need to be assessed for both potential benefits and risks. People with milder degrees of depression of relatively short duration are

\section{How this fits in}

Depression can be described at different levels of explanation and there is a tendency to adopt a dualist stance between mind and brain when thinking about treatment. This discussion article argues for a more integrated approach which allows both the dimensional nature of depression, and the

complementary nature of different treatment approaches, to be used to tailor treatment to individual needs.

likely to improve, either simply over time, or with lowintensity interventions. ${ }^{2}$ Nevertheless, patients with at least 2 months of mild to moderate depression do gain considerable benefit from antidepressants compared with usual supportive treatment, and this is probably cost-effective..$^{10}$ We would not argue that this is principally a pharmacological effect, and it may not outweigh the drawbacks of medication if less-invasive interventions are available. However, for individual patients who will not or cannot engage in other approaches, shouldn't this evidence allow at least a consideration of a trial of antidepressants?

It is rarely acknowledged that psychological interventions also pose potential risks as well as benefits. A large naturalistic study of psychological interventions in over 6000 patients seeing 71 therapists found that improvement was strongly dependent on the individual therapist, but not on the type of psychological therapy they delivered, or on the level or type of their training. ${ }^{11}$ Patients seeing the top $10 \%$ of therapists (ranked on patient outcome) were over four times more likely to recover than deteriorate (22\% and $5 \%$ respectively), whereas those seeing the bottom $10 \%$ had an equal chance of getting worse as they had of recovering $(11 \%$ in both cases).

Therefore, for psychological as well as drug treatment, the quality of the therapy given, as well as the therapeutic relationship and non-specific elements, are extremely important and treatment can cause harm as well as benefit. Drug therapy has the advantage of strict quality control, largely predictable effects, and a rigorously demonstrated specific therapeutic component compared with placebo in large numbers of people. Even the best studied psychological treatment, cognitive-behavioural therapy, has only been evaluated in a relatively small number of people and has a lack of robust evidence that it provides more than a small specific therapeutic component in rigorous trials where it has been compared with placebo or active attentional control conditions. ${ }^{12}$ Further, there is a lack of evidence that psychological therapy effects found under tightly supervised clinical trial conditions can be replicated in the routine hurly-burly of less well supervised NHS clinical practice. 


\section{PATIENT-CENTRED, NOT DOGMATIC, TREATMENT}

In putting the other side of the argument, we don't want to dismiss the value of psychological treatment. We simply argue for a more inclusive, rounded, and less ideological approach. Providing help and advice for patients with depressive disorders needs to be carried out in a spirit of negotiation, with the pros and cons of different approaches discussed in the light of each person's individual needs, clinical picture, and expectations. Middleton and Moncrieff ${ }^{1}$ argue that prescribing antidepressants may encourage patients to view themselves as helpless and 'flawed and vulnerable'. No doubt this can occur, but such problems may also occur with psychological treatment. We certainly don't advocate simply reaching for the prescription pad; in many situations the evidence that the benefits of an antidepressant will outweigh potential risks is absent, and alternatives are to be preferred. Prescription of antidepressants needs to be targeted to where it can offer a positive balance of benefits to risks: situations such as greater depression severity or duration, or a history of frequent recurrence. Importantly, the use of antidepressants doesn't preclude combination with psychological and lifestyle approaches; on the contrary, these should be encouraged. We should be open about the uncertainties inherent in treating depression, encourage a spirit of involving the patient in the decision, and help practitioners in their evaluation of the applicability of a range of therapeutic treatment approaches that may often be usefully combined, rather than simply dichotomising treatments into good or bad.

\section{Competing interests}

Ian M Anderson has received grant support and honoraria for speaking from pharmaceutical companies marketing antidepressants and chaired the Development Guideline Group for the NICE depression guideline update. Peter M Haddad has received honoraria for lecturing and consultancy and conference expenses from pharmaceutical companies marketing antidepressants. $\mathrm{He}$ has been an investigator in industry-sponsored studies of antidepressant medication.

\section{Discuss this article}

Contribute and read comments about this article on the Discussion Forum: http://www.rcgp.org.uk/bjgp-discuss

\section{REFERENCES}

1. Middleton H, Moncrieff J. 'They won't do any harm and might do some good': time to think again on the use of antidepressants? $\mathrm{Br}$ Gen Pract 2011; 61: 47-49.

2. National Institute for Health and Clinical Excellence. Depression: the treatment and management of depression in adults (update). Clinical guideline 90. London: National Institute for Health and Clinical Excellence, 2009. http://guidance.nice.org.uk/CG90/ Guidance/pdf/English (accessed 4 Nov 2010).

3. Mayberg HS, Liotti M, Brannan SK, et al. Reciprocal limbic-cortical function and negative mood: converging PET findings in depression and normal sadness. Am J Psychiatry 1999; 156(5): 675-682.

4. Elliott R, Zahn R, Deakin JF, Anderson IM. Affective cognition and its disruption in mood disorders. Neuropsychopharmacology 2010; [epub ahead of print].

5. Smith KA, Morris JS, Friston KJ, et al. Brain mechanisms associated with depressive relapse and associated cognitive impairment following acute tryptophan depletion. Br J Psychiatry 1999; 174: 525-529.

6. Kirsch I, Deacon BJ, Huedo-Medina TB, et al. Initial severity and antidepressant benefits: a meta-analysis of data submitted to the Food and Drug Administration. PLoS Med 2008; 5: e45. doi:10.1371/journal.pmed.0050045.

7. Fountoulakis KN, Moller HJ. Efficacy of antidepressants: a re-analysis and re-interpretation of the Kirsch data. Int J Neuropsychopharmacol 2010; Aug 27: 1-8. [Epub ahead of print].

8. Fournier JC, DeRubeis RJ, Hollon SD, et al. Antidepressant drug effects and depression severity: a patient-level meta-analysis. JAMA 2010; 303(1): 47-53.

9. Parker G, Anderson IM, Haddad P. Clinical trials of antidepressant medications are producing meaningless results. Br J Psychiatry 2003; 183: 102-104.

10. Kendrick T, Chatwin J, Dowrick C, et al. Randomised controlled tria to determine the clinical effectiveness and cost-effectiveness of selective serotonin reuptake inhibitors plus supportive care, versus supportive care alone, for mild to moderate depression with somatic symptoms in primary care: the THREAD (THREshold for AntiDepressant response) study. Health Technol Assess 2009; 13(22): iii-xi, $1-159$.

11. Okiishi JC, Lambert MJ, Eggett D, et al. An analysis of therapist treatment effects: toward providing feedback to individual therapists on their clients' psychotherapy outcome. J Clin Psychol 2006; 62(9): 1157-1172.

12. Lynch D, Laws KR, McKenna PJ. Cognitive behavioural therapy for major psychiatric disorder: does it really work? A meta-analytical review of well-controlled trials. Psychol Med 2010; 40(1): 9-24. 